\title{
Epidemiology of simple glaucoma and ocular hypertension
}

\author{
P. A. GRAHAM
}

University Hospital of Wales, Cardiff, and Medical Research Council Epidemiological Research Unit, Cardiff

The techniques of epidemiology originated in the study of the factors determining the spread of infectious disease. To-day, the term can be taken to mean, in a broader sense, the elucidation by the study of population samples of the natural history of disease; the determination of those factors which govern its manner of distribution; and the definition of the often elusive borderline between the normal and the diseased.

In the past 20 years, in particular, many studies have been undertaken on groups of people in an endeavour to learn more about the disease we know as simple glaucoma. Unfortunately, a first survey of the vast literature brings to light very substantial differences in prevalence in populations which are so similar genetically and environmentally that it becomes highly probable that the variations are the result largely of differences in technique and interpretation. Estimates of prevalence as high as 15.7 per cent. have been reported (Pilman, cited by Duke-Elder, 1940) and over the past 20 years the average prevalence quoted lies between I and 2 per cent.

The variability of these estimates can be explained in three ways:

(I) The samples vary in composition and few can be considered to represent a fair crosssection of the population. A number of the estimates comes from the case-finding type of survey. Here, attendance often depends on self-selection by the subject. This may or may not be desirable preventive medicine, but it is scientifically of little value to use information collected in this manner for comparative purposes.

(2) A second source of confusion is the definition of what is meant by simple glaucoma. The prevalence of tonometrically defined glaucoma-that is, pressures over $2 \mathrm{I} \mathrm{mm} . \mathrm{Hg}$ (applanation) - is many times higher than that of clinical disease with cupping of the disc and field changes, while tonographic and other methods of definition give an intermediate result.

(3) Most surveys have relied on tonometry as the first step in identifying subjects. Schwartz (1965) pointed out that small systematic errors, such as can easily result from minor differences in tonometric technique, can produce quite large variations in "positive" screening tests.

When those surveys which are suspect for epidemiological purposes are eliminated, few remain. Those which may be considered acceptable place the prevalence of clinical glaucoma, defined by the presence of tension over $21 \mathrm{~mm} . \mathrm{Hg}$, cupping of the disc, and field loss, at figures for Great Britain rather below those already quoted-for example, Hollows and Graham (1966a) 0.47 per cent., Bankes, Perkins, Tsolakis, and Wright (1968) 0.7 per cent.

These surveys, all designed to produce as accurate estimates of prevalence as possible by the exercise of care in sampling, give the most reliable assessments at present available 
of the frequency of clinical glaucoma. Many-though fewer to-day than io years ago- $\frac{0}{3}$ would consider that these criteria for the diagnosis of glaucoma are too strict, and that $\underline{\underline{z}}$ it is possible by tonometric and tonographic studies to separate glaucomatous from normal $\vec{F}$ subjects before the development of functional impairment. This brings us to the consideration of another of the functions of epidemiology in relation to chronic disease: the definition of normality.

In the case of acute illness, the dividing line between normal and diseased is reasonably $\underset{\mathbb{Q}}{\mathbb{Q}}$ well defined. In chronic disease, its definition is extremely difficult, the normal usually shading imperceptibly into the diseased. At most, one can usually say simply that, as the $\vec{\circ}$ factor one measures varies, so does the probability that the subject is in a state of pro- $\overrightarrow{\vec{H}}$ gressive disease. The range over which the change from a low to a high probability occurs is usually large, and the predictive value of an individual measurement low.

In the past 20 years or so, two overlapping phases in the study of the definition of ${ }^{\circ}$ early glaucoma can be discerned. In the first phase, emphasis was placed entirely on $\omega$ the use of tonometry to identify the disease. By the end of the first decade, culminating $\underset{\omega}{N}$ in the massive study by Leydhecker (1959), it was clear that, if the ocular tensions of ano apparently normal population were measured, the pressures observed were distributed not, $\stackrel{5}{工}$ as one might expect, symmetrically about a mean value, but with a slight excess of high $\vec{z}$ pressures (Fig. I) -an excess which can be rendered more obvious by the use of a loga-⿳亠丷厂̆ rithmic frequency scale (Fig. 2).

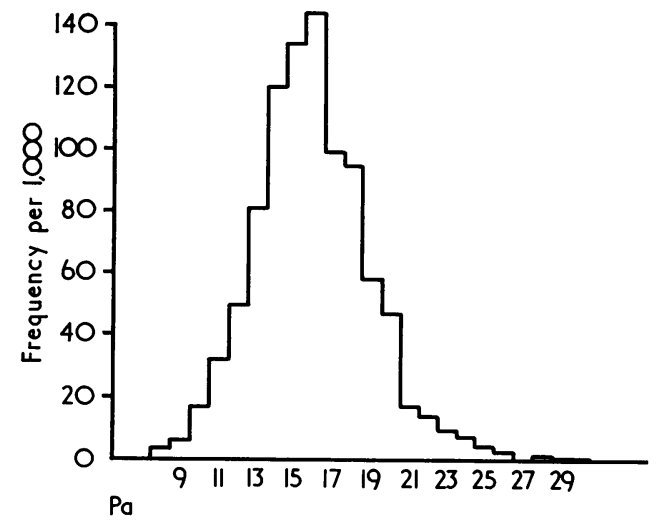

FIG. I Distribution of applanation intraocular pressure. Linear frequency scale (adapted from data of Hollows and Graham, 1966a)

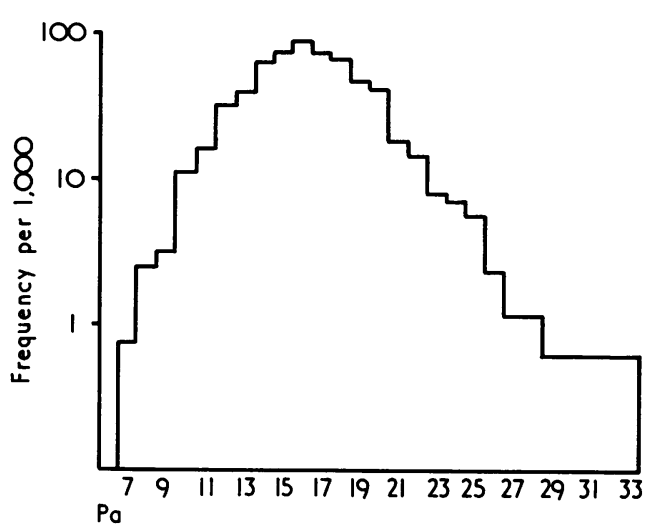

FIG. 2 Distribution of applanation intraocular pressure. Logarithmic frequency scale (adapted from $\mathrm{O}$ data of Hollows and Graham, I966a)

Most biological measurements show a symmetrical distribution. Where there is asymmetry, one might reasonably expect that our sample contains two populations, one 0 "normal" symmetrically distributed and another, which may or may not be symmetrical, N consisting of "diseased" or "abnormal" subjects. In extreme cases this may result in the c observation of a bimodal distribution.

This assumption was made in the case of intraocular pressure and, acting thereon, the $\stackrel{0}{=}$ elongated tail of the distribution was discarded and the mean and standard deviation of the $\stackrel{\mathbb{D}}{\rightarrow}$ resultant Gaussian distribution was calculated. Most observers agree that this curve has 0 a mean of approximately $16 \mathrm{~mm} . \mathrm{Hg}$ (applanation) and a standard deviation of about $\frac{\vec{D}}{\mathbb{D}}$ $3 \mathrm{~mm} . \mathrm{Hg}$. This means that a subject whose pressure exceeds $2 \mathrm{I} \mathrm{mm} . \mathrm{Hg}$ is unlikely to $\stackrel{\bigcirc}{\mathbb{D}}$ belong to the population whose pressures are symmetrically distributed.

This reasoning, on which the tonometric diagnosis of glaucoma is based, involved the 
assumption as fact what is-or was, at that time-the hypothesis that ocular tension is symmetrically distributed in non-glaurcomatous subjects. This need not necessarily be so. Davanger and Holter ( 1965 ) pointed out that ocular pressure is a function of other variables. Only if the relationship to these variables is linear would ocular pressure be distributed symmetrically even if the other variables show a Gaussian distribution. There is, however, some evidence of two populations, for Armaly (1965) and Hollows and Graham (1966a) have shown that, although in young subjects the distribution is symmetrical or nearly so, the asymmetry increases steadily after the age of 40 years.

Clearly, it is necessary to show that the bulk of subjects with pressures exceeding $2 \mathrm{I} \mathrm{mm}$. $\mathrm{Hg}$ differs from the rest of the population in some way other than the possession of a high intraocular pressure. The first attempts to achieve this end involved the use of tonographic and tonometric provocative tests. There are objections to this, however, as we may merely be measuring one of the variables of which pressure is a function. By the early i 960 s it had become increasingly obvious that the observed prevalence of clinical glaucoma, as defined by the stricter criteria of raised pressure, cupped disc, and field loss, was too low to support the hypothesis that pressures outside the Gaussian distribution led inevitably to glaucoma.

In this period also some studies were undertaken in which not only tension but also visual field studies were carried out as part of the initial screening process. These revealed the disturbing fact that field defects of a type identical to those of glaucoma could be found in subjects with pressures below $2 \mathrm{I} \mathrm{mm}$.Hg. Fig. 3 shows the distribution of such defects in one of these studies.

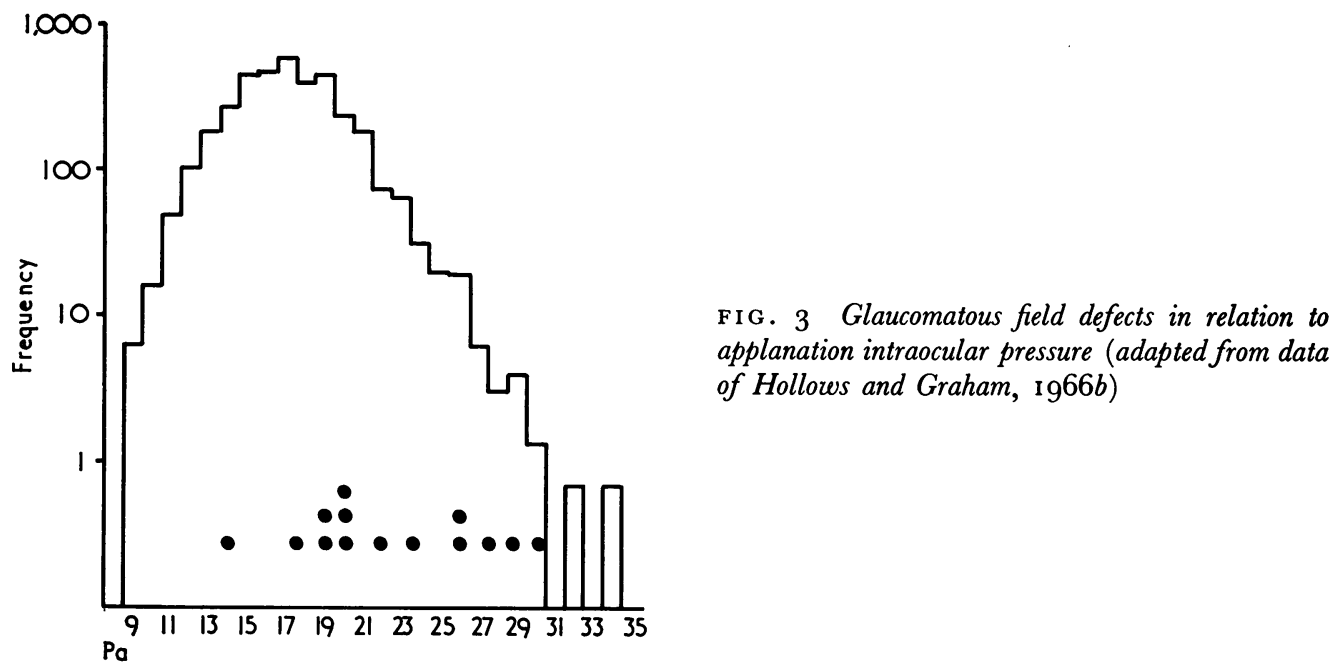

These findings made clear the need for the long-term follow-up of cohorts of what are now commonly called ocular hypertensives - that is persons with intraocular pressures exceeding $21 \mathrm{~mm} . \mathrm{Hg}$ but without functional impairment. Such studies have now been in progress for some time, and some reports have already appeared. Graham (1968) reported a follow-up study of 232 ocular hypertensives over a period of 43 months. Only one subject had developed a field defect. More recently, Armaly (I969a) reported the results of a ro-year study on subjects at all levels of tension. This also shows a very low incidence of field loss in ocular hypertensives, although with a definite tendency for defects to be more common at higher pressures. 
Tonography and water-drinking tests, it was hoped, might categorize the ocular $\frac{0}{3}$ hypertensives, presumably by separating those whose pressure was high but static and $\underline{\underline{\Xi}}$ harmless from those in whom it was rising or about to rise. Leydhecker (1966a) reported $\vec{\Rightarrow}$ that of fifty eyes with raised pressure but normal fields in 1956, 26 had developed field defects by 1963 , and that of 27 eyes which were abnormal only on tonography, five had developed defects. This sounds encouraging, but it appears that the field defects included $\frac{\bar{\omega}}{5}$ the so-called baring of the blind spot. This, it is now known, is not a defect characteristic $\stackrel{\mathbb{D}}{\mathscr{Q}}$ of early glaucoma but can be produced in normal subjects. The significance of this finding $\nRightarrow$ is therefore hard to assess.

Apart from this encouraging report, the hope that tonography would be helpful has not been realized. Graham ( 1968 ) reported that no relationship could be demonstrated ${ }_{\mathscr{O}}$ between outflow facility in 1963 and intraocular pressure in 1967, and Armaly (1969a)응. reported that tonography and the water-drinking test were no better than the initial or tension in predicting future field loss.

These follow-up studies have shown that neither simple tonometry, nor its various tonographic and provocative refinements, can clearly identify the potential glaucoma o patients in the population. All they tell us is that the higher the pressure, the greater is the probability of field loss, together with the fact that even with pressures in the upper twenties this probability is not of a very high order.

The findings which have been summarized so far lead naturally to the second phase in $\vec{\oplus}$ the use of population studies in glaucoma: the investigation of other factors which, singly or in combination, may allow the selection from the population of pre-glaucomatous subjects. The literature is again extensive, and it is only possible to summarize current trends in some of the more important lines of study.

\section{Genetic aspects}

It has long been known that there is a tendency for simple glaucoma to run in families. In the past few years, however, a considerable amount of population research has been directed towards the more precise elucidation of the mode of inheritance.

Studies of the sibs and children of glaucomatous index patients have confirmed that there is indeed a higher prevalence of clinical glaucoma in such a population-as high as 8 to 10 per cent. (Becker, Kolker, and Roth, I96o; Miller, I96 I ; Paterson, I96 I ; Leighton, I968; Jay and Paterson, I970). This is about fifteen times the prevalence in an unselected sample. It is thus obvious that either genetic or family environmental factors are at work. What is not clear from such sample figures, however, is whether the higher prevalence is the result of an increased proportion of subjects with high pressures. This point $\frac{D}{O}$ is partly clarified by several studies which show that the sibs and children of glaucoma patients have a raised mean intraocular pressure in the older age groups, and a reduced 0 outflow facility in the younger subjects (Miller, I96I; Paterson, I96I; Armaly, I965). Thus it is a reasonable hypothesis that part at least of the increased prevalence is due to an inherited tendency to a rise in tension in the later years of life.

A related field study has indicated a possible genetic basis for this hereditary trait in the analysis of the ocular pressure response to topical steroids. That such a response occurs and varies from one subject to another became evident soon after the introduction of topical steroid therapy. Becker and Mills (1963) and Armaly (1963a,b) investigated this effect in detail and showed that not only was there a greater proportion of hypertensive responses in glaucomatous subjects, but also that the distribution of response in the population showed evidence of consisting of three sub-populations. 
One explanation for this finding is that the three groups may be genetically determined. Further investigation (Becker and Ballin, I965; Armaly, I966a; Becker and Chevrette, I966) showed that this is probably the case. To summarize the findings, it is evident that the three levels of response can reasonably be considered to represent genotypes corresponding to the combinations of two genes designated either as " $\mathrm{n}$ " and "g" or "Pl" and "Ph." Those homozygous for " $n$ " or "Pl" form a low response group, those for " $g$ " or "Ph" a high response group, and heterozygotes occupy an intermediate position.

Forewarned by the error of equating ocular hypertension with glaucoma, it is necessary to consider carefully what this means. It has been shown only that the pressure response of an eye to the administration of topical steroids is genetically determined, and that the gene " $\mathrm{g}$ " or " $\mathrm{Ph}$ " is more prevalent in patients with clinical glaucoma and, as a corollary, in their relatives.

Initially it was thought that the "gg" homozygous state represented genetic glaucoma. This is certainly not true. Clinical glaucoma occurs in heterozygotes and even in " $n$ " homozygotes (Armaly, I967a) and the discrepancy between the observed prevalence of high responders and clinical glaucoma suggests that follow-up studies would be likely to demonstrate that only a proportion of high responders progress to clinical glaucoma.

Another possibility to be considered is that those whose tension is predestined to rise in later life can be distinguished in youth by steroid provocation. This seems quite possible, but again caution must be observed in accepting hypothesis as fact, especially bearing in mind the lack of correlation between outflow facility and subsequent change in tension.

We can, however, now assume with reasonable confidence that a tendency to ocular hypertension is inherited, and that the steroid response may identify the presence of one of the genes responsible. Ocular hypertension is not glaucoma, however, and the greatly increased prevalence of clinical glaucoma in sibs and children of glaucoma patients seems more than is likely to result simply from an increased frequency of higher pressures. It is evident that other factors, probably genetic though possibly also acquired, must be involved, and that the disease simple glaucoma is the result of multifactorial transmission. To put this another way, the glaucomatous state develops when a subject occupies an unfavourable position in the tail of not one, but several distributions, some at least of which are genetically determined.

There is evidence to support this in the studies of the high prevalence of phenylthiourea non-tasters in glaucomatous subjects (Becker and Morton, I964) and possibly also in the observations that the size of the optic cup and even applanation pressure show evidence of multifactorial inheritance (Armaly 1967c,d, I969a, r97ob; Armaly and Sayegh, 1969).

Racial differences in the prevalence of glaucoma are known to exist (Mann, 1966), although accurate estimates of prevalence are few. It seems probable that the varying prevalences relate mainly if not wholly to genetic differences, as no relevant environmental factors have been identified. If factors other than pressure alone, whether genetic or environmental, are involved in the genesis of clinical glaucoma, one would expect comparative studies to show that the relative prevalences of ocular hypertension and glaucoma are not constant in comparisons between populations of different races. In the studies of Hollows and Graham (1966a) and Wallace and Lovell (1969), accurate comparison is possible, as the techniques and criteria are common to both. In the Ig66 study, on a population of predominantly Welsh origin, a prevalence of 6.8 per cent. for ocular hypertension was associated with 0.47 per cent. clinical glaucoma, while on a Jamaican population of West African Negro origin, the corresponding figures are 4.7 per cent. ocular hypertension and I.4 per cent. clinical glaucoma. This difference suggests that the probable 
increased prevalence of glaucoma in the Negro may be due to factors other than increased ocular pressure.

\section{Diabetes and glaucoma}

The observation of some connection between diabetes mellitus and simple glaucoma goes back over 40 years when Grafe (1924) observed that proliferative retinopathy is less common in cases of diabetes with raised tension. Further investigation has shown somewhat conflicting results. Becker (197I) has found that both simple glaucoma and is ocular hypertension are more common in diabetes and also that the prevalence of overt $\vec{\circ}$ diabetes and positive glucose tolerance tests in glaucoma patients is about three times $\vec{\omega}$ that of a normal population. Further, his studies show that the frequency of clinical glaucoma relative to ocular hypertension is increased in diabetes, suggesting greater sus- $\frac{0}{0}$. ceptibility apart from any association between diabetes and ocular hypertension.

The latter may be at least partly due to a common genetic background, as studies have shown an apparent relationship between the response to dexamethasone provocation, $N$

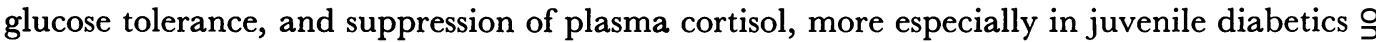
(Levene and Schwartz, I968; Armaly, r967e; Becker and Ramsey, I970; Kolker, Stewart, Alton, and LeMon, 197I).

There is some conflicting evidence, however, as it was reported by Gavey (1966), 宁 that diabetes was conspicuous by its rarity in a survey of 300 glaucomatous patients, and $\vec{\omega}$ more recently by Bouzas, Gragoudas, Balodimos, Brinegar, and Aiello (197 I), that the $N$ mean intraocular pressure of diabetics did not differ significantly from that of the normal population. Armaly ( $1967 \mathrm{e}$ ) also reported that the parents of juvenile diabetics failed to show the expected excess of the $\mathrm{Ph}$ gene on steroid provocation.

The hypothesis that the diabetic is more prone to the visual field changes of glaucoma and that proliferative retinopathy occurs less frequently in the hypertensive eye is also not conclusively proven. For example, Christiansson (196r, r 965) reported that, although there was some apparent resistance of glaucomatous diabetics to proliferative retinopathy, the position is complicated by the fact that in diabetics inflow, as measured by suction-cup methods, is low and falls markedly with the onset of proliferative retinopathy. It seems clear that the relation between these two diseases is one of considerable interest and promise, but one about which there is still much to be learned.

\section{Glaucoma and blood pressure}

Arterial hypertension may be slightly more common in glaucomatous subjects, but it is doubtful if the association is meaningful (Duke-Elder, r969a). Recent studies on patients who present visual field defects have shown that it is more probable that a factor of con- N siderable importance is the relation between intraocular pressure and blood pressure in the narrow sense of capillary perfusion pressure in the optic nerve head. Drance, N Wheeler, and Pattullo (1968), in comparing the two eyes of patients in whom only one had marked visual field change, found that in the affected eye the difference between 0 the diastolic central artery pressure and intraocular pressure was in most cases significantly less than in the unaffected eye, while in those cases where there was a higher pressure? difference, there was usually a history of an episode of illness likely to have been associated 0 with marked hypotension.

Related studies (Lobstein and Herr, 1966; Drance, 1962) have also demonstrated $\frac{?}{\mathbb{D}}$ that the effects on the visual field of elevation of the intraocular pressure are related to diastolic ophthalmic artery pressure. 
Also of interest is the observation of Suda and Abe (1964) that the glaucoma-like scotomata produced by bulbar compression appear at lower intraocular pressures than in normal subjects. These observations do much to explain why simple tonometry and related tests have failed to provide an accurate index of the incidence of clinical glaucoma.

To summarize the present position, it has been established by population studies that simple glaucoma is a disease determined in the individual by a number of factors, the measurement of none of these being in itself sufficient to categorize the subject as normal or abnormal. As information from these studies accumulates, we are beginning to obtain more accurate estimates of the probability of the development of clinical glaucoma requiring treatment, but as yet our knowledge is insufficient to allow us to diagnose with confidence the patient in whom the premonitory signs indicate the inevitability of deteriorating visual function if treatment is not instituted.

There is, however, reasonable hope that such a goal is attainable, although the techniques involved may not be simple and will therefore be expensive-a fact of considerable relevance to the public health administrator who is approached for support in screening programmes.

\section{COMMENTARY}

\section{OGULAR HYPERTENSION AND POPULATION SURVEYS}

The concept of ocular hypertension is unhelpful and the intraocular pressure must always be correlated with the appearance of the discs. The highest intraocular pressure without field changes in the Cardiff survey was 34 and in the Bedford survey $29 \mathrm{~mm} . \mathrm{Hg}$. In the Bedford survey only two out of I $3^{8}$ persons with ocular hypertension (intraocular pressure 19 to $23 \mathrm{~mm}$. Hg) developed field changes in a 5-year period, but two out of twelve with suspicious discs developed field changes in this time. Re-examination after 5 years of over 500 persons with normal tensions and normal discs revealed one case of raised tension with glaucomatous field defects. One of the major parameters is the change in pressure with time, about which very little is known.

In Armaly's population studies, four persons developed glaucoma during the follow-up period and three out of four of these would not have been spotted if the cut-off point had been 21 or 22 $\mathrm{mm} . \mathrm{Hg}$ intraocular pressure. They were all in their twenties when the field defect was discovered and had other lesions which could have been spotted, such as abnormal blood sugar or vascular changes. The intraocular pressure levels in these four patients were by no means the highest in the population studied.

\section{Some factors in the production of low tension glaucoma}

\section{STEPHEN M. DRANCE}

From the Department of Ophthalmology, University of British Columbia, The Glaucoma Unit, Vancouver General Hospital, and the D.V.A. Hospital Shaughnessy, Vancouver B.C., Canada

The enigma of low tension glaucoma has existed since von Graefe (1857a) described "amaurosis with excavation" which had all the ophthalmoscopic features of chronic simple glaucoma without elevation in intraocular pressure (Schnabel, 1908). It must be noted, however, that the intraocular pressure in his time was checked by digital tonometry. Von Graefe withdrew the concept, probably at the instigation of Donders, who believed 